\title{
Simulation of AVC Measurements
}

\author{
M. Rottinger, N. Seifert ${ }^{\dagger}$, and S. Selberherr \\ Institute for Microelectronics, TU Vienna \\ Gusshausstr. 27-29, A-1040 Vienna, Austria \\ tDigital Semiconductor \\ 75 Reed Road, Hudson, MA 01749, USA
}

\begin{abstract}
The recently developed Auger Voltage Contrast (AVC) method is an electronic probing technique for rapid delineation of pn-junctions. The simulation of AVC measurements can improve the understanding of the involved physics and facilitate the development of procedures for automatic extraction of the junction position from measurement data. This paper describes the simulation of AVC measurements using a device simulator.
\end{abstract}

\section{Introduction}

Development of new semiconductor devices and the down scaling of proven designs increases the demands on accurate positioning of the doping and creates a need for new methods to measure the doping distribution. One of the recently developed electronic properties probing techniques is the Auger Voltage Contrast (AVC) method [1].

In the AVC method a beam of high energetic electrons is focused on the surface of the test device. The kinetic energy of emitted secondary electrons is measured. The measured energy is a function of the surface potential which is influenced by the doping in the device.

AVC measurements are quite time consuming and need specialized equipment. Therefore simulation of such measurements is a valuable tool for the development and improvement of procedures for automatic delineation of the pn-junction and extraction of doping distributions from the measurement data and to gain better understanding of the involved physical effects.

\section{The AVC Method}

In the AVC method a beam of high energetic electrons is focused on the surface of a cross-sectioned test device. The device is connected by a contact to ground voltage. The incident electrons generate electron-hole pairs in the semiconductor and a fraction of the secondary electrons has enough kinetic energy to leave the semiconductor.

The built-in potential causes a band bending which is a function of the doping. The kinetic energy of the emitted secondary electrons is measured and compared to the energy of electrons emitted from an undoped semiconductor. The difference in the 
observed kinetic energy corresponds to the band bending caused by the doping, the presence of surface states, and the interaction of the probing electron beam with the device.

The shift in the energy of the secondary electrons can be considerably reduced by a dipole layer at the surface of the semiconductor caused by surface states and the connected potential. Theoretical studies [1] indicate that this effect is only important for small beam currents. Very small beam currents have to be avoided in actual measurements because the number of emitted secondary electrons which can be used for averaging is too small to produce reliable results.

An important property of the AVC method is that the locations $x_{\mathfrak{i}}$ where the electron beam impinges on the semiconductor surface and $x_{\mathrm{e}}$ where the potential is extracted are varied during a scan. Therefore it is not sufficient to look for the location where the second derivative of the potential vanishes to determine the pn-junction position. The second derivative of the extracted potential is a function of the charge caused by the doping $\rho^{\text {dop }}$ and the charge from the injected electrons and the generated electron-hole pairs $\rho^{\text {inj. }}$.

$$
\nabla^{2} \varphi=f\left(\rho^{\mathrm{dop}}\left(x_{\mathrm{e}}\right), \rho^{\mathrm{inj}}\left(x_{\mathrm{i}}, x_{\mathrm{e}}\right)\right)
$$

The zero of the second derivative of the potential is located at the metallurgical junction only if $\rho^{\text {inj }}$ can be neglected. For higher beam currents the zero location shifts considerably from the junction and some kind of inverse modeling is necessary to determine the exact location of the junction.

\section{Extension of MINIMOS-NT}

Simple analytical approximations describing the interaction of the electron beam and the semiconductor break down for beam current densities used in real measurements. Therefore the semiconductor equations have to be solved numerically to calculate the surface potential. The device simulator MINIMOS-NT [2] has been enhanced to be capable of simulating such measurements.

Appropriate models were added to the simulator to account for the injection of electrons by the electron beam and the electron-hole pair generation caused by the incident high energy electrons. The effects of surface states have been neglected because they are important only for small beam currents.

The distribution of the injected primary electrons and the generated secondary carriers in lateral and vertical direction were modeled by Gauß distributions [3]. The distance of the maximum of the distribution from the surface and the lateral and vertical standard deviations are functions of the energy of the incident electrons. Estimates for the distance of the maximum from the surface and the vertical and lateral standard deviations were taken from Monte Carlo simulations performed by the program SESAME [4].

The most important parameters of the new models are the electron beam current and the average number of electron-hole pairs generated per incident electron because they determine the number of secondary carriers. The exact distribution of the secondary carriers has only small influence on the surface potential because the standard deviations are small compared to the diffusion length of the carriers which typically are of the order of several ten $\mu \mathrm{m}$.

The simulation of an AVC scan requires a number of simulations of the same device with varying locations of the injected electrons and of the generated electron-hole 


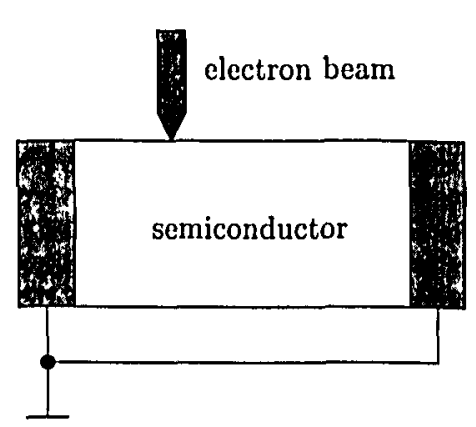

Figure 1: Geometry of the simulated device.

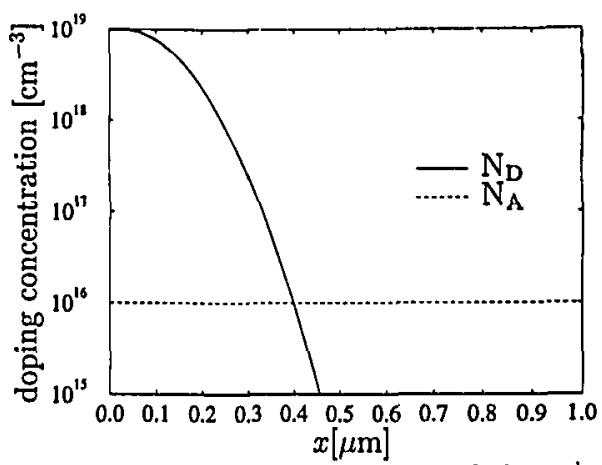

Figure 2: Doping distribution of the $\mathrm{n}^{+} \mathrm{p}$ diode. The metallurgical junction is located at $x=0.4 \mu \mathrm{m}$.

pairs. From the result of each simulation the surface potential is extracted at the location of the electron beam.

\section{Simulation of a $n^{+} p$ Diode}

As an example a $\mathrm{n}^{+} \mathrm{p}$ diode has been simulated. The simulated device consisted of a block of silicon with side contacts to ground level (see Fig. 1). A constant acceptor background doping of $\mathrm{N}_{\mathrm{A}}=10^{16} \mathrm{~cm}^{-3}$ and a varying donor doping with a maximum value of $N_{D}=10^{19} \mathrm{~cm}^{-3}$ was used. The metallurgical junction was located at $x=0.4 \mu \mathrm{m}$. Fig. 2 shows the doping distribution of the device. A constant electron energy of $3 \mathrm{keV}$ was assumed for all calculations and the beam current was varied between $10 \mathrm{pA}$ and $1 \mathrm{nA}$.

The resulting surface potential is plotted in Fig. 3. On the lower doped side of the pn-junction the vast number of secondary carricrs causes a shift in the potential compared to the built-in potential. The number of secondary carriers is proportional to the beam current, therefore the potential shift shows a strong dependence on the beam current.

For beam currents up to $500 \mathrm{pA}$ the potential drop across the pn-junction is quite large and the slope is steepest near the junction. For $\ln A$ beam current the potential drop decreases to approximately one fifth of the value for the built-in potential and the slope is very flat and nearly constant in the vicinity of the junction. The decrease in the total potential drop across the pn-junction and in the slope set an upper limit for experimentally feasible beam currents.

Fig. 4 shows the second derivative of the surface potential. The zero of the second derivative is not located at the metallurgical junction and shifts when the magnitude of the electron beam current or the primary electron energy is varied.

\section{Conclusion}

Simulations show that for high beam currents some sort of inverse modeling is necessary for proper delineation of the pn-junction. Simulation of AVC measurements has proven a capable tool for developing methods for pn-junction extraction and an important aid in interpreting the results of AVC measurements. 


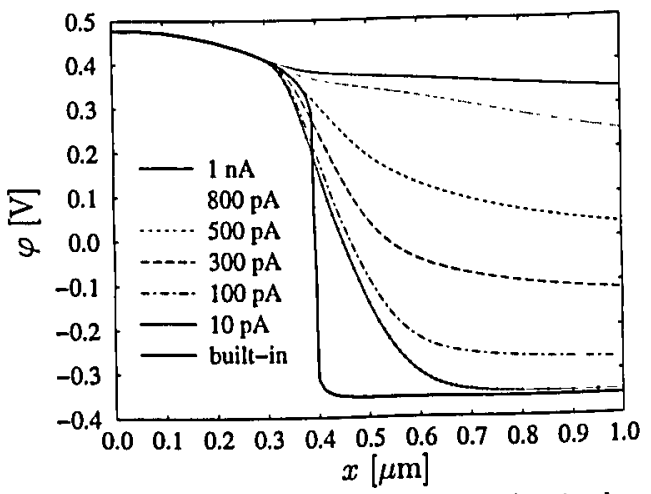

Figure 3: Surface potential of $\mathrm{a}^{+} \mathrm{p}$ diode.

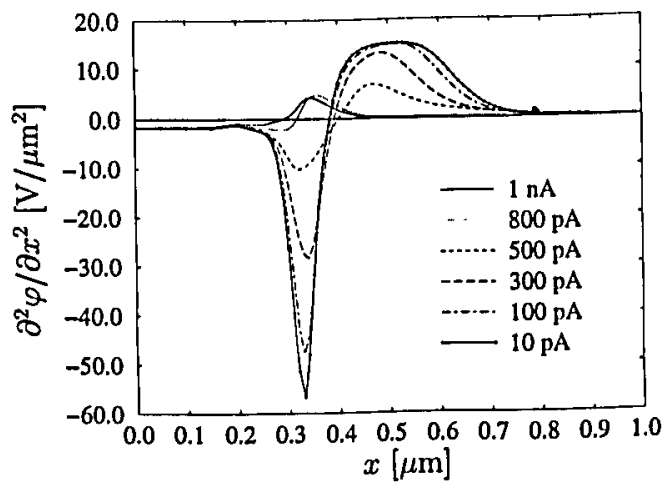

Figure 4: Second derivative of the surface potentiala $n^{+} p$ diode.

\section{Acknowledgment}

This work is supported by the "Christian Doppler Forschungsgesellschaft", Vienna, Austria.

\section{References}

[1] W. Werner, H. Lakatha, H. Smith, L. LeTarte, V. Ambrose, and J. Baker, "Auger Voltage Contrast Imaging for the Delineation of 2-Dimensional Junctions in Crosssectioned MOS Devices," J.Vac.Sci.Technol., accepted for publication 1998.

[2] T. Simlinger, H. Kosina, M. Rottinger, and S. Selberherr, "MINIMOS-NT: A Generic Simulator for Complex Semiconductor Devices," in 25th European Solid State Device Research Conference (H. de Graaff and H. van Kranenburg, eds.), pp. 83-86, 1995.

[3] D. Kyser and D. Wittry, "Spatial Distribution of Excess Carriers in Electron-Beam Excited Semiconductors," Proc.IEEE, pp. 733-734, May 1967.

[4] H. Wagner, A. Pfeiffer, C. Schiebl, and W. Werner, "Monte Carlo Simulation of Electron Scattering for Arbitrary 2D Structures Using a Modified Quadtree Geometry Discretization.," Microchim. Acta, vol. 13, p. 533, 1995. 